[microreview]

Diamond Open Access

\title{
A right zero semigroup is left-cancellative
}

\author{
Open Mathematics Collaboration*广
}

June 28, 2020

\begin{abstract}
We prove the proposition addressed in the title of this paper.
\end{abstract}

keywords: semigroup, right zero, left-cancellative, abstract algebra

The most updated version of this paper is available at https://osf.io/g6snd/download

\section{Notation \& Definition}

1. $[1,2]$

2. $\mathcal{S}=$ semigroup

3. $\forall x, y, z \in \mathcal{S}:(z x=z y) \rightarrow(x=y) \quad$ left-cancellative

4. $\forall x, y \in \mathcal{S}: x y=y \quad$ right zero semigroup

*All authors with their affiliations appear at the end of this paper.

†Corresponding author: mplobo@uft.edu.br | Join the Open Mathematics Collaboration 


\section{Proposition}

5. A right zero semigroup is left-cancellative. [1]

\section{Proof}

6. Suppose $z x=z y$ for an arbitrary $z$.

7. From (4) and (6), $z x=x$ and $z y=y$.

8. From (6) and (7), $x=y$.

9. From (6) and (8), since $z$ is arbitrary,

$$
\forall x, y, z \in S:(z x=z y) \rightarrow(x=y) .
$$

10. Therefore, from (3) and (9), $\mathcal{S}$ is left-cancellative.

\section{Final Remarks}

11. $(\forall x, y \in \mathcal{S}: x y=y) \rightarrow(\forall x, y, z \in \mathcal{S}:(z x=z y) \rightarrow(x=y))$

\section{Open Invitation}

Review, add content, and co-author this paper [3,4]. Join the Open Mathematics Collaboration (https://bit.ly/ojmp-slack). Send your contribution to mplobo@uft.edu.br.

\section{Open Science}

The latex file for this paper together with other supplementary files are available [5]. 


\section{Ethical conduct of research}

This original work was pre-registered under the OSF Preprints [6], please cite it accordingly [7]. This will ensure that researches are conducted with integrity and intellectual honesty at all times and by all means.

\section{References}

[1] Cain, Alan J. Nine Chapters on the Semigroup Art. AJC Porto \& Lisbon, 2020. http://www-groups.mcs.standrews.ac.uk/\%7ealanc/teaching/m431/

[2] Lobo, Matheus P. "Open Mathematics Knowledge Base." OSF Preprints, 13 May 2020. https://doi.org/10.31219/osf.io/evq8a

[3] Lobo, Matheus P. "Microarticles." OSF Preprints, 28 Oct. 2019. https://doi.org/10.31219/osf.io/ejrct

[4] Lobo, Matheus P. "Simple Guidelines for Authors: Open Journal of Mathematics and Physics." OSF Preprints, 15 Nov. 2019. https://doi.org/10.31219/osf.io/fk836

[5] Lobo, Matheus P. "Open Journal of Mathematics and Physics (OJMP)." OSF, 21 Apr. 2020. https://doi.org/10.17605/osf.io/6hzyp

[6] COS. Open Science Framework. https://osf.io

[7] Lobo, Matheus P. "A Right Zero Semigroup Is Left-cancellative." OSF Preprints, 27 June 2020. https://doi.org/10.31219/osf.io/g6snd 


\section{The Open Mathematics Collaboration}

Matheus Pereira Lobo (lead author, mplobo@uft.edu.br) $)^{1,2}$

${ }^{1}$ Federal University of Tocantins (Brazil); ${ }^{2}$ Universidade Aberta (UAb, Portugal) 